\title{
SPECTROPHOTOMETRIC DETERMINATION OF OILS WITH RADICALS OF CONJUGATED OCTADECATRIENOIC ACIDS
}

\begin{abstract}
Anh Van Nguyen, V. I. Deineka, ${ }^{*}$ and L. A. Deineka
UDC 543.428:543.851

An approach specific for triacylglycerides (TAGs) is proposed for chromatographic separation of compounds with different chromophores using spectrophotometric detection instead of preliminary calibration of the detector using unavailable (or difficultly accessible) reference standards. A wavelength similar to an isosbestic point (278 $\mathrm{nm}$ ) is found for this approach for TAGs containing radicals of three isomeric conjugated octadecatrienoic acids (COAs) and can be used to determine the ratio of the amounts of TAGs with isomeric radicals without introducing correction factors for the sensitivity of each compound. A mathematical procedure for processing UV spectra of TAGs containing COA radicals is proposed for finding these points. The specifics of the TAG composition allow the isolation and use of highly labile pure compounds to be avoided. The method is developed using seed oils of red valerian (Centranthus $D C$.), TAGs of which are formed from two isomeric COAs ( $\alpha$-and $\beta$-eleosteric), and pomegranate (Punica granatum L.) with radicals of another pair of COAs (punicic and $\alpha$-eleostearic) as examples.
\end{abstract}

Keywords: seed oils, triacylglycerides, Centranthus, Punica granatum, Momordica charantia, UV spectrum, HPLC, $\alpha$-eleostearic acid, $\beta$-eleostearic acid, punicic acid, analogs of isosbestic points.

Introduction. Vegetable oils containing triacylglycerides (TAGs) with conjugated octadecatrienoic acids (COAs) are unique among plant oils because of their high biological activity [1]. Electronic spectroscopy is convenient for studying such oils because the conjugation causes a bathochromic shift of the $\pi \rightarrow \pi^{*}$-transitions (as compared with the transition in monoenoic compounds) that is proportional to the number of conjugated double bonds in the chromophore [2]. Vibronic structure is observed in the electronic spectrum of conjugated trienoic acids in the central strongest band (transition from the ground vibrational state of a lower electronic level into the first excited vibrational state of the first excited electronic level) in the range $267-275 \mathrm{~nm}$. Only four stereoisomers are found in nature although eight cis-trans-isomers can exist for trienoic higher fatty acids with the unsaturated bonds localized along the carbon chain. This happens because COA derivatives in which the central double bond has only the trans-configuration are observed in plant sources [3]. Also, only two different positions of the double bonds along the carbon chain occur in the natural isomers, i.e., with double bonds on atoms $8,10,12$ and $9,11,13$.

Changing the cis-configuration to the trans-configuration in the conjugated chain of double bonds is known to produce a hypsochromic shift of the absorption maximum [3]. The stereoisomeric acids can have only three different spectra for the three different configurations of the conjugated double bonds in the chromophore because the orientation of the chromophore relative to a nonconjugated carboxylic-acid or ester group has no effect (Fig. 1). Thus, radicals of several isomeric COAs can be present in several oils [4]. Quantitative determination of their contents in samples using spectrophotometric detection and areas under the curves is hindered if their spectral characteristics differ [5].

The goal of the present work was to use the specific TAG compositions to determine the ratio of extinction coefficients of punicic and $\alpha$ - and $\beta$-eleostearic acids when they were both present in TAGs using spectral characteristics of compounds separated by HPLC with diode-array detection.

Experimental. Oils were obtained by extraction with $n$-hexane of seeds of Centranthus (varieties Mauve Ringing and Plasma Seeds), Momordica charantia (grown in Belgorod in 2015), and pomegranate (Punica granatum purchased

* To whom correspondence should be addressed.

The National Research University "Belgorod State University," Institute of Engineering Technologies and Natural Sciences, 85 Pobeda Str., Belgorod, 308015, Russia; email: deineka@bsu.edu.ru. Translated from Zhurnal Prikladnoi Spektroskopii, Vol. 85, No. 3, pp. 474-479, May-June, 2018. Original article submitted December 7, 2017. 


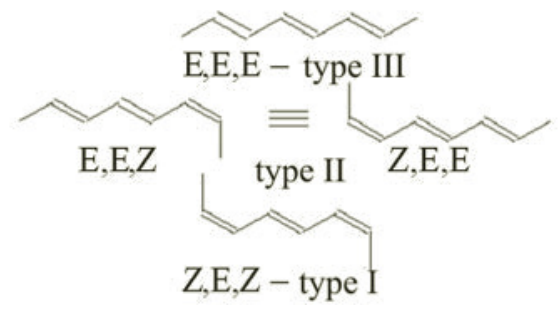

Fig. 1. Structures of chromophores in conjugated octadecatrienoic higher fatty acids.

at a fruit market in 2017). Chromatograms were recorded on an Agilent Infinity 1200 chromatograph with a diode-array detector for recording spectra in the detector cuvette and a Kromasil 100-5C18 chromatography column $(250 \times 4.6 \mathrm{~mm})$. The mobile phase (solvent for recording spectra) consisted of $i-\mathrm{PrOH}$ (35 vol.\%) and $\mathrm{MeCN}(65$ vol.\%) at flow rate $1 \mathrm{~mL} / \mathrm{min}$. The column temperature was $30^{\circ} \mathrm{C}$. Chromatograms were also recorded using a Waters RI 401 refractometric detector and a mobile phase consisting of $\mathrm{MeCN}$ (30 vol.\%) and $\mathrm{Me}_{2} \mathrm{CO}$ (70 vol.\%). The Agilent ChemStation 32 program was used to control the chromatograph, record spectra and chromatograms, and store and process information. Spectra exported from this program were also processed using the Excel program. Optical densities in the range 245-295 nm with measurement uncertainties $<0.002$ were used in the calculations. The Magicplot Student 2.7.2 program was used to separate overlapping peaks in the chromatograms.

Results and Discussion. Figure 2 shows chromatograms of seed oils from Centranthus, M. charantia, and P. granatum. The TAG peaks in chromatograms [6,7] and electronic spectra of Centranthus and M. charantia oils could be assigned by comparing retention times and electronic spectra as the tri- $\alpha$-eleostearate $(\alpha \mathrm{E})_{3}(1 \mathrm{c})$, di- $\alpha$-eleostearate-linoleate $(\alpha \mathrm{E})_{2} \mathrm{~L}(2), \alpha$-eleostearate-dilinoleate $\alpha \mathrm{EL}_{2}(3)$, di- $\alpha$-eleostearate-oleate $(\alpha \mathrm{E})_{2} \mathrm{O}(4), \alpha$-eleostearate-linoleate-oleate $\alpha \mathrm{ELO}$ (5), $\alpha$-eleostearate-linoleate-palmitate $\alpha \operatorname{ELP}(6)$, di- $\alpha$-eleostearate-stearate $(\alpha \mathrm{E})_{2} \mathrm{~S}(7)$, and $\alpha$-eleostearate-linoleate-stearate $\alpha$ ELS (8). The TAG peaks of Centranthus seed oil grouped under the number 2 were strongest and most interesting. The corresponding compounds were isomers according to differences in their electronic spectra (Fig. 3).

As noted above, only three types of chromophores occur in natural conjugated trienoic higher fatty acids (Fig. 1). The first type, in which three $\mathrm{C}=\mathrm{C}$ bonds are conjugated in the cis-trans-cis configuration $(Z, E, Z)$, occurred in punicic $\left(18: 3^{9 Z 11 E 13 Z}\right)$ and jacaric acids $\left(18: 3^{8 Z 10 E 12 Z}\right)$ [8], which had different spectra and the greatest wavelength at the absorption maxima according to our data. Changing one of the two end cis-bonds to a trans-bond should produce four (until now only three were observed in nature) new isomeric acids with identical electronic spectra [8], i.e., 18:3 ${ }^{9 Z 11 E 13 E}$ or $\alpha$-eleostearic, $18: 3^{9 E 11 E 13 Z}$ or catalpic acid, $18: 3^{8 Z 10 E 12 E}$ that is not found in nature, and $18: 3^{8 E 10 E 12 Z}$ or calendic acid. This substitution led to hypsochromic shifts of all absorption maxima. The hypsochromic shifts were even greater on going to the fully transconfiguration. However, electronic spectra were obtained experimentally only for TAG with 18:39E11E13E or $\beta$-eleostearic acid. Electronic spectra of TAG containing only 18:3 ${ }^{8 E 10 E 12 E}$, which was observed in TAG of calendula oil [9], were problematical to record because of its low content.

The results agreed with the literature [10], according to which seed oil of Centranthus contained simultaneously $\alpha$ - and $\beta$-eleostearic acids. In this instance, peaks $2,2 \mathrm{a}$, and $2 \mathrm{~b}$ represented compounds in which two $\alpha$-eleostearic acids were sequentially replaced by $\beta$-eleostearic acids, i.e., di- $\alpha$-eleostearate-linoleate $(\alpha \mathrm{E})_{2} \mathrm{~L}(2), \alpha$-eleostearate- $\beta$-eleostearate-linoleate $\alpha E \beta E L(2 a)$, and di- $\beta$-eleostearate-linoleate $(\beta E)_{2} L(2 b)$. Figure 3 shows the normalized spectra. Because the compound for peak 2a was a mixed TAG with an exact 1:1 ratio of conjugated trienoic acids, the ratio of extinction coefficients of the two isomeric radicals could be exactly determined. If the normalized spectrum of $(\alpha \mathrm{E})_{2} \mathrm{~L}, F_{\alpha \mathrm{E}}(\lambda)$, was added to the normalized spectrum of $(\beta \mathrm{E})_{2} \mathrm{~L}, F_{\beta E}(\lambda)$, with correction factor $g$, then the normalized spectrum should coincide with the normalized experimental spectrum of $\alpha E \beta E L$. The position of the absorption maximum $\lambda_{\max }$ required for this was determined from the experimental spectrum $F_{\alpha \mathrm{E} \beta \mathrm{EL}}(\lambda)$ :

$$
F_{\alpha \mathrm{E} \beta \mathrm{E} \text { theor }}(\lambda) \equiv\left[F_{\alpha \mathrm{E}}(\lambda)+g F_{\beta \mathrm{E}}(\lambda)\right] /\left[F_{\alpha \mathrm{E}}\left(\lambda_{\max }\right)+g F_{\beta \mathrm{E}}\left(\lambda_{\max }\right)\right] .
$$

Coefficient $g$ was chosen according to the mean least-square deviation between the calculated and experimental spectra $F(g)$ in which the summation covered all wavelengths (optical densities were recorded in 1-nm steps):

$$
F(g)=\Sigma\left[F_{\alpha \mathrm{E} \beta E L \exp }(\lambda)-F_{\alpha \mathrm{E} \beta E L} \text { theor }(\lambda)\right]^{2} .
$$




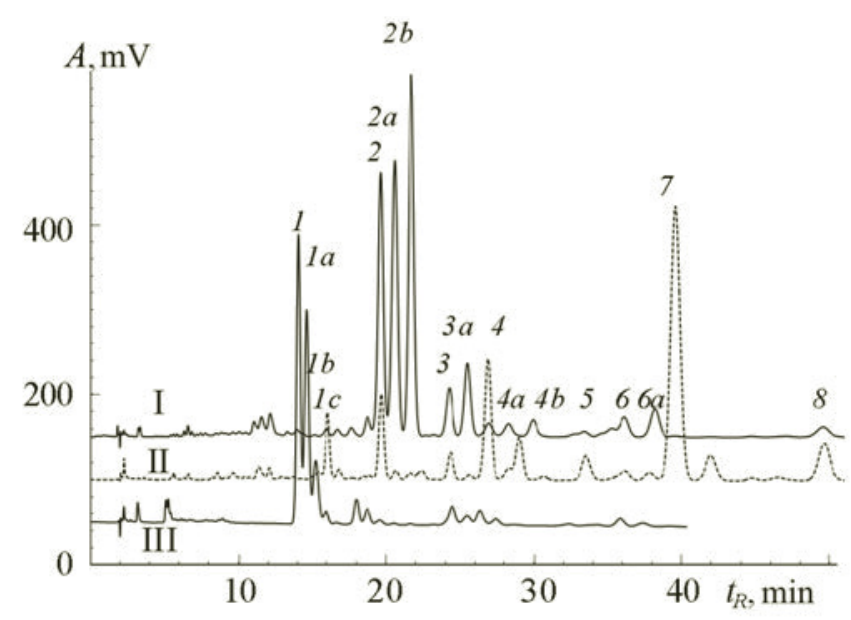

Fig. 2. Separation of TAGs from three seed oils: Centranthus (I), Momordica charantia (II), and Punica granatum (III).

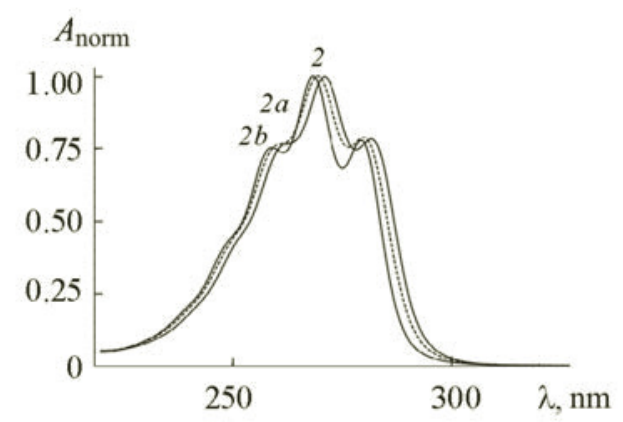

Fig. 3. Electronic absorption spectra of $\mathrm{E}_{2} \mathrm{~L}$ TAGs; radical $\mathrm{E}=\alpha$ - or $\beta$-eleostearic acid; $(\alpha \mathrm{E})_{2} \mathrm{~L}(2), \alpha \mathrm{E} \beta \mathrm{EL}(2 \mathrm{a})$, and $(\beta \mathrm{E})_{2} \mathrm{~L}(2 \mathrm{~b})$ (numbers correspond to peaks in Fig. 2); solvent, $\mathrm{CH}_{3} \mathrm{CH}(\mathrm{OH}) \mathrm{CH}_{3}\left(35\right.$ vol.\%) $+\mathrm{CH}_{3} \mathrm{CN}(65$ vol.\%).

Calculations using several different sets of spectra (with different mobile phases) showed that the minimum of function $F(g)$ corresponded to $\beta=1.005 \pm 0.020$. The resulting ratio was considerably less than the experimental data for the isomeric acids themselves [5] that were obtained for solutions in a different solvent (cyclohexane). However, the problems associated with the unknown purity of the samples used to determine the ratio of their extinction coefficients were eliminated in our instance. Therefore, another problem could be solved, i.e., calculation of the ratio of the amounts of the various TAG species using spectrophotometry to determine the areas under the curves. Areas under peaks obtained for TAG containing $\beta$-eleostearic acid or a mixture of both acids should be corrected by introducing the appropriate correction factors if the chromatogram was recorded at a wavelength corresponding to one of the absorption maxima of $\alpha$-eleostearic acid. In our instance, the problem could be solved more simply by recording in a single plot the normalized spectrum of TAG with only $\alpha$-eleostearic acid and the spectrum of TAG with only $\beta$-eleostearic acid but with the previously found coefficient $g$. Points equivalent to isosbestic points could be found (Fig. 4). Figure 4 shows five such points at $\lambda=260.5,263.4,269.8,278.0$, and $280.5 \mathrm{~nm}$. Wavelength $280.5 \mathrm{~nm}$ was most convenient and could also be used to record chromatograms and to determine directly areas under curves without any correction factors.

The proposed method for calculating areas under peaks was validated by comparing results obtained for three spectrophotometric procedures, i.e., from absorption maxima for $\beta$ - and $\alpha$-eleostearates, for the isosbestic point analog proposed above, and with refractometric detection (Table 1). The refractive indices of all three isomeric TAGs should be approximately equal. In fact, the third spectrophotometric procedure produced results that were closest to those with refractometric detection.

The ratio of extinction coefficients for TAGs with punicic and $\alpha$-eleostearic acids were found in a similar manner. For this, a chromatogram of pomegranate seed oil in which the first peak corresponded to the tripunicate $\mathrm{Pu}_{3}$ and the second, 


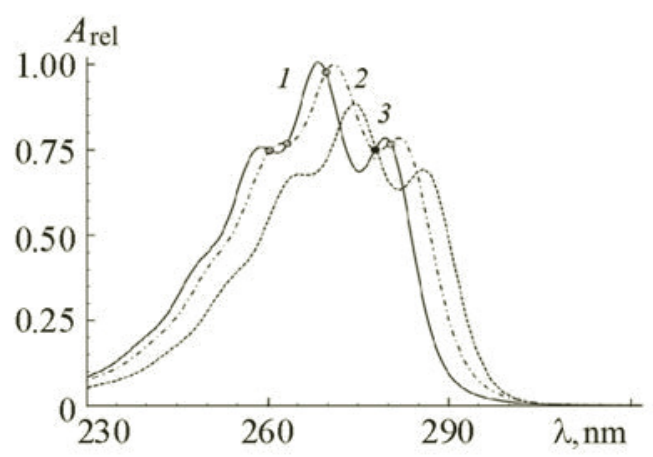

Fig. 4. Absorption spectra of TAGs containing $\alpha$ - (2) and $\beta$-eleostearic (1) and punicic acid radicals (3) with correction factors.

TABLE 1. Relative Amounts of Three Isomeric TAGs from Centranthus Seed Oil Calculated in Four Ways

\begin{tabular}{|c|c|c|c|c|}
\hline \multirow{2}{*}{ TAG } & \multicolumn{3}{|c|}{ Spectrophotometry } & \multirow{2}{*}{ Refractometry } \\
\cline { 2 - 5 } & $\lambda=269 \mathrm{~nm}$ & $\lambda=271 \mathrm{~nm}$ & $\lambda=280 \mathrm{~nm}$ & \\
\cline { 2 - 4 } & \multicolumn{3}{|c|}{ Fraction from areas under curves in total isomeric TAGs, mol\% } \\
\hline$(\alpha \mathrm{E})_{2} \mathrm{~L}$ & 28.2 & 29.3 & 30.6 & 30.0 \\
$(\alpha \mathrm{E})(\beta \mathrm{E}) \mathrm{L}$ & 31.6 & 31.7 & 31.9 & 32.9 \\
$(\beta \mathrm{E})_{2} \mathrm{~L}$ & 40.29 & 39.0 & 37.5 & 37.1 \\
\hline
\end{tabular}

TABLE 2. Areas under Curves of Four Main Peaks in Chromatogram of Pomegranate Seed Oil Calculated in Three Ways

\begin{tabular}{|c|c|c|c|}
\hline \multirow{2}{*}{ TAG } & \multicolumn{2}{|c|}{ Spectrophotometry } & \multirow{2}{*}{ Refractometry } \\
\cline { 2 - 4 } & $\lambda=271 \mathrm{~nm}$ & $\lambda=285 \mathrm{~nm}$ & 47.0 \\
$\mathrm{Pu}_{3}$ & 44.7 & 47.8 & 38.0 \\
$\mathrm{Pu}_{3}(\alpha \mathrm{E})$ & 38.96 & 37.9 & 12.6 \\
$\mathrm{Pu}(\alpha \mathrm{E})_{2}$ & 13.35 & 11.9 & 2.4 \\
$(\alpha \mathrm{E})_{3}$ & 2.94 & 2.4 & \\
\hline
\end{tabular}

to mixed TAG $\mathrm{Pu}_{2}(\alpha \mathrm{E})$, and the $(\alpha \mathrm{E})_{2} \mathrm{~S}$ peak from a chromatogram of M. charantia oil were used (Fig. 2). In this instance, the calculation formulas were slightly changed to take into account the number of acid radicals. The normalized spectrum of $\mathrm{Pu}_{3}, F_{\mathrm{Pu} 3}(\lambda)$, multiplied by 2 was combined with the normalized spectrum of $(\alpha \mathrm{E})_{2} \mathrm{~S}, F_{(\alpha \mathrm{E}) 2 \mathrm{~S}}(\lambda)$, with correction factor $g$. Normalization should produce a spectrum coinciding with the normalized experimental spectrum of $\mathrm{Pu}_{2}(\alpha \mathrm{E})$. The position of the absorption maximum $\lambda_{\max }$ that was required for normalization was also determined from the experimental spectrum $F_{\mathrm{Pu} 2(\alpha \mathrm{E})}(\lambda)$ :

$$
F_{\mathrm{Pu} 2(\alpha \mathrm{E}) \text { theor }}(\lambda) \equiv\left[2 F_{\mathrm{Pu}}(\lambda)+g F_{(\alpha \mathrm{E}) 2 \mathrm{~S}}(\lambda)\right] /\left[2 F_{\mathrm{Pu} 3}\left(\lambda_{\max }\right)+g F_{(\alpha \mathrm{E}) 2 \mathrm{~S}}\left(\lambda_{\max }\right)\right] .
$$

Coefficient $g$ was chosen according to the mean least-square deviation between absorptions in the calculated and experimental spectra in which the summation covered all wavelengths:

$$
F(g)=\Sigma\left[F_{\mathrm{Pu} 2(\alpha \mathrm{E}) \exp }(\lambda)-F_{\mathrm{Pu} 2(\mathrm{aE}) \text { theor }}(\lambda)\right]^{2} .
$$

In this instance, an unexpectedly relatively large parameter $g(1.15 \pm 0.05)$ was obtained. This was indicative of a significant difference in the extinction coefficients of punicic and $\alpha$-eleostearic acids although the difference for these acids was reported to be minimal [3]. 
Analogs of isosbestic points were determined in the same manner. The wavelength of one of them $(285 \mathrm{~nm})$ was used to record chromatograms for direct calculation of the ratio of amounts of the various TAGs. The results were similar to those with refractometric determination of TAGs (Table 2), indicating that the estimated ratio of extinction coefficients for TAGs containing punicic and $\alpha$-eleostearic acids was correct. Superimposition of the three spectra with correction factors showed that one point was close to an isosbestic analog for all three types of electronic spectra, i.e., $\lambda \sim 278 \mathrm{~nm}$ (Fig. 4). Therefore, this wavelength could be recommended for recording chromatograms of any oils containing all known fatty COAs, including their arbitrary mixtures, and for calculating their relative contents without introducing correction factors.

Conclusions. A method for determining the ratios of extinction coefficients of TAGs containing three different COAs was proposed and used electronic absorption spectra of the TAGs recorded in the cuvette of an HPLC diode-array detector during their separation. The effectiveness of using wavelengths at analogs of isosbestic points to calculate the relative amounts of TAGs from areas under peaks without introducing correction factors was demonstrated. A universal wavelength $(278 \mathrm{~nm})$ that was suitable for analysis of oils with any combination of known isomeric COAs using direct calculations and areas under peaks without introducing correction factors was found.

\section{REFERENCES}

1. G.-F. Yuan, X.-E. Chen, and D. Li, Food Funct., 5, 1360-1368 (2014); DOI: 10.1039/c4fo00037d.

2. E. G. Hammond and W. O. Lundberg, J. Am. Oil Chem. Soc., 30, 433-438 (1953); https://doi.org/10.1007/BF02641673.

3. C. Y. Hopkins and M. J. Chisholm, J. Am. Oil Chem. Soc., 45, 176-182 (1968); https://doi.org/10.1007/BF02915346.

4. T. Takagi and Y. Itabashi, Lipids, 16, 546-551 (1981); https://doi.org/10.1007/BF02535054.

5. J. S. Hoffmann, R. T. O'Connor, D. C. Heinzelman, and W. G. Bickford, J. Am. Oil Chem. Soc., 34, 338-342 (1957); DOI: $10.1007 / \mathrm{BF} 02638280$.

6. M.-K. Chang, E. J. Conkerton, D. C. Chapital, P. J. Wan, O. P. Vadhwa, and J. M. Spiers, J. Am. Oil Chem. Soc., 73, 263-265 (1996); https://doi.org/10.1007/BF02523907.

7. A. V. Turtygin, V. I. Deineka, and L. A. Deineka, Zh. Anal. Khim., 68, 619-624 (2013) [A. V. Turtygin, V. I. Deineka, and L. A. Deineka, J. Anal. Chem., 68, 558-563 (2013); DOI: 10.1134/S1061934813060142].

8. V. I. Deineka, Sorbtsionnye Khromatogr. Protsessy, 5, No. 4, 507-517 (2005).

9. F. V. Dulf, D. Pamfil, A. D. Baciu, and A. Pintea, Chem. Cent. J., 7, 8 (2013); DOI: 10.1186/1752-153X-7-8.

10. A. P. Tulloch, Lipids, 17, 544-550 (1982); http://doi.org/10.1007/BF02535382. 